\title{
Do early HER2-overexpression breast cancer patients benefit from undergoing neoadjuvant trastuzumab and mastectomy? A meta-analysis
}

This article was published in the following Dove Press journal: Cancer Management and Research

\author{
Lin $\mathrm{He}^{\mathrm{l}, *}$ \\ Qian $\mathrm{Wu}^{\mathrm{l}, *}$ \\ Jing Xiong ${ }^{2}$ \\ Zhumin $\mathrm{Su}^{3}$ \\ Biyuan Zhang 4 \\ Yuhua Song' \\ 'Breast Center B Ward, The Affiliated \\ Hospital of Qingdao University, Qingdao, \\ Shandong Province, People's Republic of \\ China; ${ }^{2}$ Department of Geriatrics, The \\ Affiliated Hospital of Qingdao University, \\ Qingdao, Shandong Province, People's \\ Republic of China; ${ }^{3}$ Department of \\ Neurology, The People's Hospital of China \\ Medical University, Shenyang, Liaoning \\ Province, People's Republic of China; \\ ${ }^{4}$ Department of Radiotherapy, The Affiliated \\ Hospital of Qingdao University, Qingdao, \\ Shandong Province, People's Republic of \\ China
}

*These authors contributed equally to this work
Correspondence: Yuhua Song Breast Center B ward, The Affiliated Hospital of Qingdao University, No. 59 Haier Road, Laoshan District, Qingdao, Shandong Province, People's Republic of China

Tel +86 I 369869 I275

Email qdsongyh@I26.com
Purpose: To assess the overall survival (OS) of early human epidermal growth factor receptor 2 (HER2)-enriched breast cancer patients after receiving neoadjuvant trastuzumab (NAT) compared to adjuvant trastuzumab (AT) treatment and the difference in local-regional relapse (LRR) rate with this tumor and treatment between women after mastectomy and women after breast-conserving therapy (BCT).

Methods: Articles were retrieved from PubMed, Embase, Web of Science, and Cochrane Library. A pooled odds ratio (OR) with a $95 \%$ confidential interval (CI) was calculated. The StataSE version 12.0 software was employed for meta-analysis.

Results: Twelve available clinical studies containing 2366 subjects were included. The OS of NAT compared with that of AT was not significantly different (pooled OR=1.04; 95\% CI, 0.47-2.33). There was a significantly lower LRR rate for patients with mastectomy compared to those with BCT (pooled OR $=0.58$; 95\% CI, 0.38-0.89); however, subgroup analysis revealed that the significant advantage of LRR for mastectomy compared to BCT was only represented in women without trastuzumab treatment (pooled $\mathrm{OR}=0.52 ; 95 \% \mathrm{CI}, 0.31-0.88$ ) compared to those who received trastuzumab treatment (pooled $\mathrm{OR}=0.71 ; 95 \% \mathrm{CI}, 0.34-1.49$ ).

Conclusion: Early stage HER2-overexpression breast cancer patients benefit with an equivalent OS from NAT treatment compared to AT. Patients who underwent mastectomy and BCT experienced a similar LRR rate if they received anti-HER2 targeted therapy of trastuzumab, but the LRR rate was discernibly reduced in patients who received mastectomy compared to BCT if they did not also receive trastuzumab treatment.

Keywords: human epidermal growth factor receptor 2, trastuzumab, mastectomy, breast conserving therapy, breast cancer

\section{Introduction}

Neoadjuvant systematic chemotherapy in breast cancer is a preoperative treatment, which is initially applied locally to advanced tumors to downgrade primary tumor and regional lymph nodes and enable their removal by operation. Currently, neoadjuvant chemotherapy is widely used in early resectable disease, mainly to allow breastconserving therapy (BCT). ${ }^{1-3}$ Adjuvant chemotherapy is postoperative and attenuates breast cancer mortality by at least $15 \%{ }^{4}$ Many randomized clinical trials and metaanalyses confirm the equivalent overall survival (OS) of breast cancer women after receiving preoperative chemotherapy compared to those who undergo postoperative chemotherapy; ${ }^{5-9}$ however, these studies are imperfect, as they all failed to perform the subgroup analysis on different molecular subtypes of breast cancer. 
In the period before 1980, the modified radical mastectomy was the standard surgical method for breast cancer. In the early 1980s, two published randomized trials reformed this standard modality and confirmed that the survival benefits from BCT were equivalent to those of mastectomy. ${ }^{10,11}$ The Early Breast Cancer Trialists' Collaborative Group carried out a large-scale meta-analysis that further pooled the results from both randomized trials, finding the equality of OS and disease-free survival (DFS) between mastectomy and BCT. ${ }^{12}$ Currently, instead of mastectomy, $\mathrm{BCT}$ has become the appropriate and preferred treatment for most early stage breast cancer patients, ${ }^{13}$ and it prevents the physical and psychological burden of sacrificing breast on women as much as possible. ${ }^{14}$

Human epidermal growth factor receptor 2 (HER2)-overexpression breast cancer accounts for approximately 15$25 \%$ of the primary breast tumors. ${ }^{15}$ Trastuzumab is currently indispensable for treating HER2-positive breast cancer. Prior to treatment with the anti-HER2 targeted drug, the local-regional recurrence (LRR) of this cancer molecular subtype is significantly greater than that of other phenotypes of disease, specifically Luminal A. ${ }^{16}$ Voduc et a ${ }^{16}$ found that the LRR rate of HER2-enriched breast cancer patients without trastuzumab treatment who underwent BCT and those who received trastuzumab was $21 \%$ and $17 \%$ at 10 years, respectively. After receiving trastuzumab, there is discernible reduction in the high LRR rate of HER2-positive breast tumors; a study from Debled et $\mathrm{al}^{17}$ showed that those women treated with BCT or mastectomy had a significantly reduced 4-year LRR rate of $2.9 \%$ or $0 \%$, respectively. However, the LRR rates between these two surgical strategies in treating HER2-amplified breast carcinoma were not compared in these studies. Therefore, the aim of our article was to separately pool all clinical studies that concomitantly documented the OS outcomes of early HER2-overexpression breast cancer patients who received neoadjuvant trastuzumab (NAT) and adjuvant trastuzumab (AT) and that concurrently described the LRR rate of those women undergoing mastectomy and BCT. We aimed to identify the more applicable and preferably primary treatment strategy for this subtype of breast tumor. We included women treated with NAT or mastectomy as the study cohort and those with AT or BCT treatment as the control cohort.

\section{Methods}

\section{Search strategy}

Based on the PRISMA-IDP Statement, ${ }^{18}$ electronic searches were performed in PubMed, Embase, Web of Science, and
Cochrane Library using the following retrieval strategy: ((HER2 OR (Human epidermal growth factor receptor 2)) AND ((“Breast Neoplasms"[Mesh]) OR (Breast cancer) OR (Breast tumour) OR (Breast tumour) OR (Breast carcinoma) OR (Breast Neoplasm))) AND ((((Neoadjuvant therapy) OR (Neoadjuvant treatment)) AND ((Adjuvant therapy) OR (Adjuvant treatment)) AND (Trastuzumab OR Herceptin) AND (Overall Survival)) OR (Mastectomy AND ((Breast conservation surgery) OR (Breast conserving surgery) OR (Breast preservative surgery) OR (Breast preservation surgery) OR (Breast preserving surgery) OR (Breast preservative surgery) OR (Breast conservation therapy) OR (Breast conserving therapy) OR (Breast preservative therapy) OR (Breast preservation therapy) OR (Breast preserving therapy) OR (Breast preservative therapy) OR (Breast conservation treatment) OR (Breast conserving treatment) OR (Breast preservative treatment) OR (Breast preservation treatment) OR (Breast preserving treatment) OR (Breast preservative treatment)) AND ((Local-regional relapse) OR (Local-regional recurrence) OR (Local relapse) OR (Local recurrence)))). No restrictions were required during the retrieval. The searching of citation was terminated as of 28th May 2019.

\section{Inclusion criteria}

- Early HER2-enriched breast cancer patients;

- English publications which covered the number or the rate of event and the total sample size;

- Clinical trials which concurrently documented the data of LRR after BCT and that after mastectomy or OS after NAT and that after AT. LRR referred to the first recurrence of tumor in the ipsilateral breast, the chest wall or regional lymphatics, without evidence of distant metastasis. The definition of BCT was a combination of radiotherapy and surgery strategy involving lumpectomy, segmental mastectomy, quadrantectomy, or wedge resection. The methods of mastectomy included simple mastectomy and modified radical mastectomy. OS ranged from the date of diagnosis to the date of death or loss to follow-up.

\section{Exclusion criteria}

- Trials published in non-English;

- Patients with moderate-to-advanced stage disease;

- Male patients;

- Reviews, case reports, and conference papers;

- Other details that did not meet the inclusion criteria. 
Two co-authors (Qian Wu and Jing Xiong) independently screened the retrieved citations and reserved the pertinent studies according to the titles, abstracts, and full-text articles. If some disagreements surfaced, they were resolved by the third reviewer (Zhumin $\mathrm{Su}$ ).

\section{Data abstraction}

The following information was abstracted using Microsoft Excel version 2016 (Microsoft Corporation, Redmond, Washington, USA) by two authors (Jing Xiong and Zhumin Su): first author, publication year, study duration, original nation, median age, median follow-up, whether patients received trastuzumab treatment, the number of patients undergoing BCT or mastectomy and how many of them developed LRR, and the number of women who received NAT or AT and how many died from either treatment paradigms. Provided that there were any inconsistencies, they were resolved by discussion.

\section{Statistical analysis}

The included clinical studies were discussed by their differences of eligible criteria, chemotherapy strategy, and implementation of radiotherapy, as well as the risk of bias of study evaluation using the new version of the Cochrane tool. The crude odds ratio (OR) with its $95 \%$ confidence interval (CI) of LRR with respect to mastectomy compared to BCT or OS regarding NAT versus AT in each trial was calculated and then pooled together. If the event number was not shown in the publication, it was computed based on the end point percentages of LRR and OS or other information. A heterogeneity $\mathrm{Chi}^{2}$ test with a significance level of $P<0.1$ was used to estimate the heterogeneity among different studies; ${ }^{19}$ when it was not significant $(P>0.1)$, a fixed-effect Mantel-Haenszel model was utilized to pool the data, otherwise, a random-effect Mantel-Haenszel model was applied. ${ }^{19}$ The publication bias was assessed by creating a Begg's funnel plot with 95\% CI and Egg's test with a significance level of $P<0.05$. All statistical tests were performed in StataSE version 12.0 (Stata Corporation, College Station, TX, USA).

\section{Results}

\section{Search results}

After systematic retrieval in the databases described earlier, 3078 citations were obtained. After deleting the duplicates ( $\mathrm{n}=903)$, citations categorized as reviews $(\mathrm{n}=244)$, conference papers $(n=702)$, and case reports $(n=34), 1195$ records were remained for the title and abstract screening. An additional 1139 citations were removed by this step, and 56 articles qualified for full-text article assessment; we excluded the nonmatched documents that were reviews $(n=8)$ or covered non-HER2-overexpression breast tumors $(\mathrm{n}=9)$ as well as those with no valid control cohort $(\mathrm{n}=12)$ or no prognosis $(n=15)$. Ultimately, 12 satisfactory studies ${ }^{16,17,20-23}$ were involved in the meta-analysis. The PRISMA flow diagram of selecting qualified clinical trials is outlined in Figure 1.

\section{Characteristics of included studies}

The original nations of the included articles were USA $(n=2)$, France $(n=1)$, Canada $(n=3)$, the Netherlands $(n=1)$, Spain $(n=1)$, the UK $(n=1)$, India $(n=1)$, China $(n=1)$, and Japan $(n=1)$. The sample size ranged from 43 to 748 (median: 81.5), with a total number of 2366 subjects. The year range of included studies was 2008 to 2018 . The other details including study duration, whether trastuzumab was received or absent, chemotherapy strategy, and which cohort (study cohort or control cohort) received radiotherapy are presented in Table 1 .

\section{Meta-analysis results}

Four eligible trials with 960 subjects were included to analyze the OS between NAT and AT. As shown in Figure 2, the pooled data indicated that there was no significant difference in the OS of HER2-overexpressing breast tumors after receiving NAT compared to AT (pooled $\mathrm{OR}=1.04 ; 95 \% \mathrm{CI}, 0.47-2.33$ ).

There were 8 valid studies with 1406 patients who were collected to investigate the comparison of the LRR rate between mastectomy and BCT in treating HER2-amplified breast cancer. The data analysis suggested that women with this tumor undergoing mastectomy benefited from a lower LRR rate than those in the treatment of BCT (pooled $\mathrm{OR}=0.58 ; 95 \%$ CI, 0.38-0.89) (Figure 3A). We further divided the included trials into subgroups based upon whether trastuzumab administration to patients was documented. Interestingly, the subgroup with absent trastuzumab still showed a lower LRR rate with mastectomy than with BCT (pooled OR $=0.52 ; 95 \%$ CI, 0.31-0.88), while the LRR rate between these two surgical interventions was not significantly different in the subgroup that received trastuzumab (pooled OR $=0.71 ; 95 \%$ CI, 0.34-1.49) (Figure 3B).

\section{Risk of bias in analyzed studies}

All included trials referencing the OS of NAT versus AT and the LRR of mastectomy versus BCT were combined to judge 


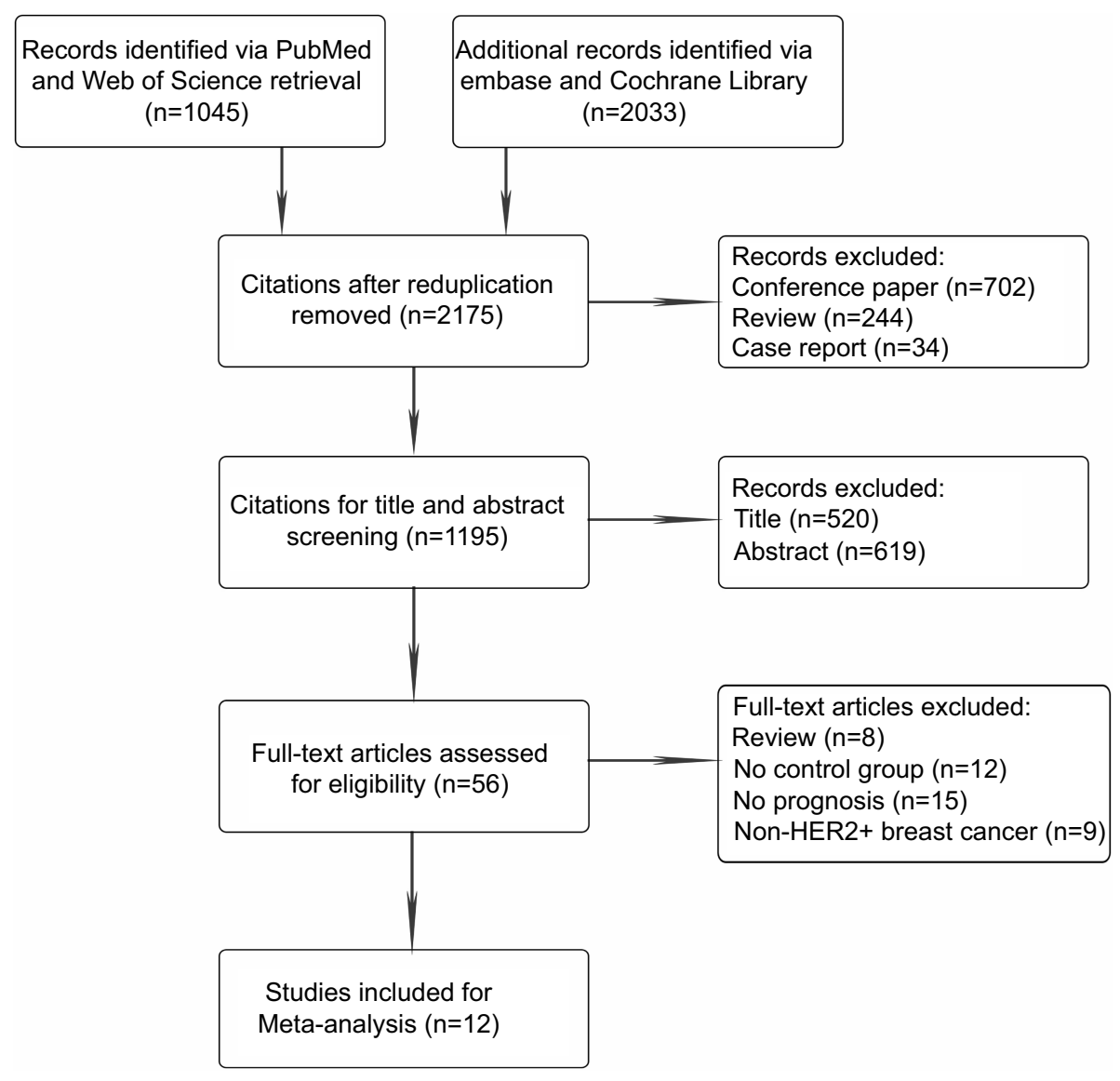

Figure I The study selection procedure.

Abbreviation: HER2, human epidermal growth factor receptor 2.

each risk of bias domain. The risk of bias summary and the risk of bias graph are presented in Figure 4A and B, respectively.

\section{Publication bias}

In the Begg's funnel plots of the OS of NAT versus AT and the LRR of mastectomy versus BCT, data were uniformly arranged above and below the axis. Consistently, their publication bias in light of Egg's test was not statistically significant ( $p=0.844$ and 0.515 , respectively), suggesting that the moderate heterogeneity in the OS of NAT versus AT was not by virtue of bias (Figure S1 and S2 in Supplementary, page 1 and 2).

\section{Discussion}

HER2-positive breast cancer necessitates at least 1 year of continuous trastuzumab treatment to achieve optimal therapeutic efficacy. Our results demonstrate that the early stage HER2-enriched breast tumor patients who receive NAT have a comparative OS to those who undergo AT.
Additionally, greater local-regional control is attained in those who receive mastectomy compared to those who receive BCT before the era of trastuzumab administration; nevertheless, women with this disease who receive trastuzumab treatment derive an equivalent LRR rate with the two surgical approaches.

In agreement with our results, some trials ascertain no differences between patients with early HER2-positive breast cancer following treatment with NAT compared to AT in the local relapse-free survival, event-free survival, relapse-free survival as well as breast cancer-specific survival. $^{24-26}$ However, the study of Chatterjee et $\mathrm{al}^{24}$ indicated that the DFS of patients in AT cohort was significantly longer than that in NAT cohort. Trastuzumab emtansine (T-DM1) is an antibody-cytotoxic drug conjugate of trastuzumab and the cytotoxic agent emtansine; it has efficacious antitumor effects in trastuzumab-sensitive and trastuzumab-resistant HER2-amplified breast tumors and was initially approved for advanced HER2-positive breast cancer patients who have previously received 


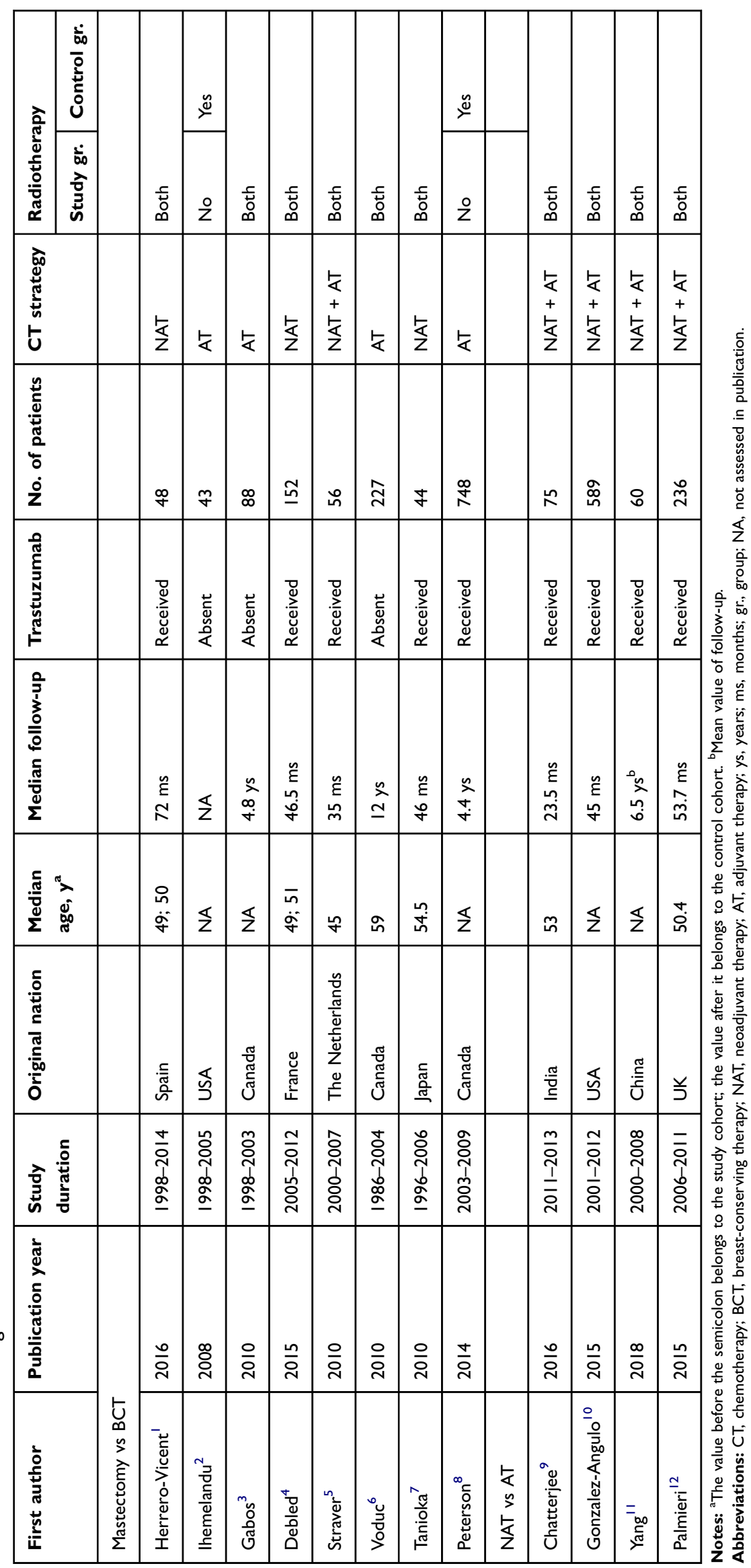




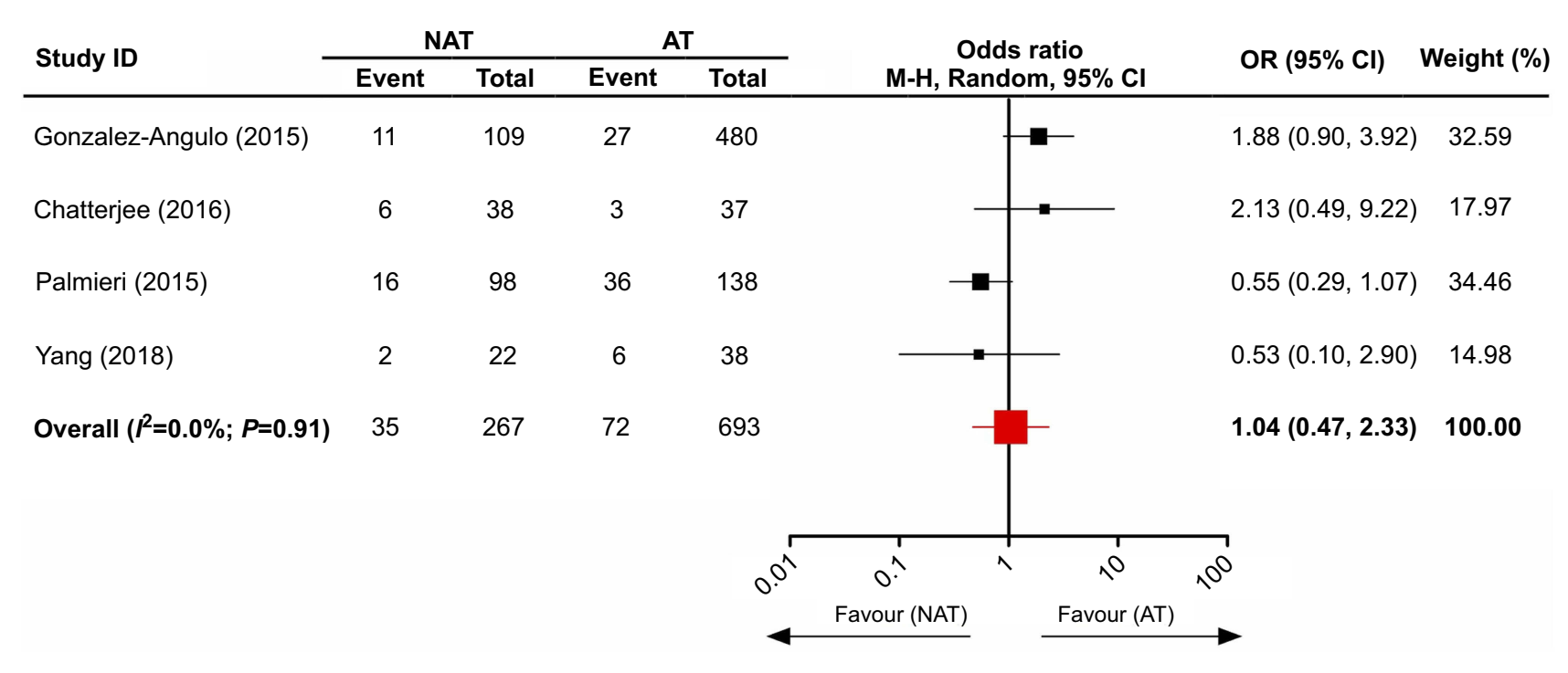

Figure 2 The comparison of overall survival between neoadjuvant trastuzumab and adjuvant trastuzumab. Abbreviations: NAT, neoadjuvant trastuzumab; AT, adjuvant trastuzumab.

trastuzumab treatment. ${ }^{27,28}$ The articles of Hurvitz ${ }^{29}$ and Minckwitz, ${ }^{28}$ respectively, assessed the tumor response and the survival prognosis of early stage HER2-enriched subtype of breast tumors after treatment with T-DM1 and trastuzumab. Their findings demonstrated that women with this disease subset who received T-DM1 had significantly superior DFS and distant relapse-free survival and reduced pathological complete response ( $\mathrm{pCR}$ ) rates compared to those who received trastuzumab, but the OS was not significantly different. The mechanisms of action of T-DM1 eradicating HER2-positive breast tumor foci contrast to those of trastuzumab. T-DM1 activates caspase-3/caspase-7 to induce apoptotic cell death and releases the intracellular enzyme adenylate kinase which contributes to the cellular lysis. Trastuzumab antagonizes the constitutive growth-signaling properties of the HER2 system, mobilizes immune cells to kill the tumor target, and reinforces chemotherapy-induced cytotoxicity. ${ }^{27,30}$

For breast cancer patients with the HER2-enriched subtype, there are two distinct eras determined by the usage of trastuzumab in the adjuvant setting. In the period preceding the use of anti-HER2 targeted therapy, the LRR rate of this tumor ranges from $4 \%$ to $15 \% .^{16,31}$ The natural process of this phenotype of breast cancer has been positively influenced by trastuzumab. ${ }^{32}$ Yin et $\mathrm{al}^{33}$ analyzed six relevant studies and indicated that trastuzumab reduced the LRR rate of HER2-positive breast cancer by $50 \%$. This conclusion was supported by a retrospective study performed by Panoff and colleagues, ${ }^{34}$ which found that the LRR rate of women with this disease who underwent mastectomy plus trastuzumab was $1.7 \%$. Our article finds the different LRR rates between HER2-amplified breast tumors undergoing mastectomy and those undergoing BCT before the application of trastuzumab coupled with the identical LRR rate among both surgical scenarios after its administration, which mirrors the trastuzumab-adjusted alteration of the natural course of this disease.

There is a viewpoint suggesting that the prognosis of HER2-enriched breast cancers primarily depends on the biological characteristics of the disease rather than the content of surgical approach. ${ }^{35}$ Nevertheless, it may be somewhat confined and not comprehensive. A study enrolled 618 breast cancer patients that underwent either BCT or mastectomy, 92 of whom were classified as the HER2 subtype. ${ }^{21}$ In the BCT cohort, HER2 subtype of breast cancer and lymph node positivity were independent prognostic factors associated with high-risk of LRR. This cancer subset was not associated with increased risk of LRR in the mastectomy cohort. Similarly, our results reaffirm that the different extents of surgery may be related to the prognosis of HER2-overexpression breast carcinoma devoid of trastuzumab treatment.

There are some limitations of this article that deserve mention. First, despite the absence of publication bias, only English literature was included which might give rise to selection bias. Second, the included studies in 


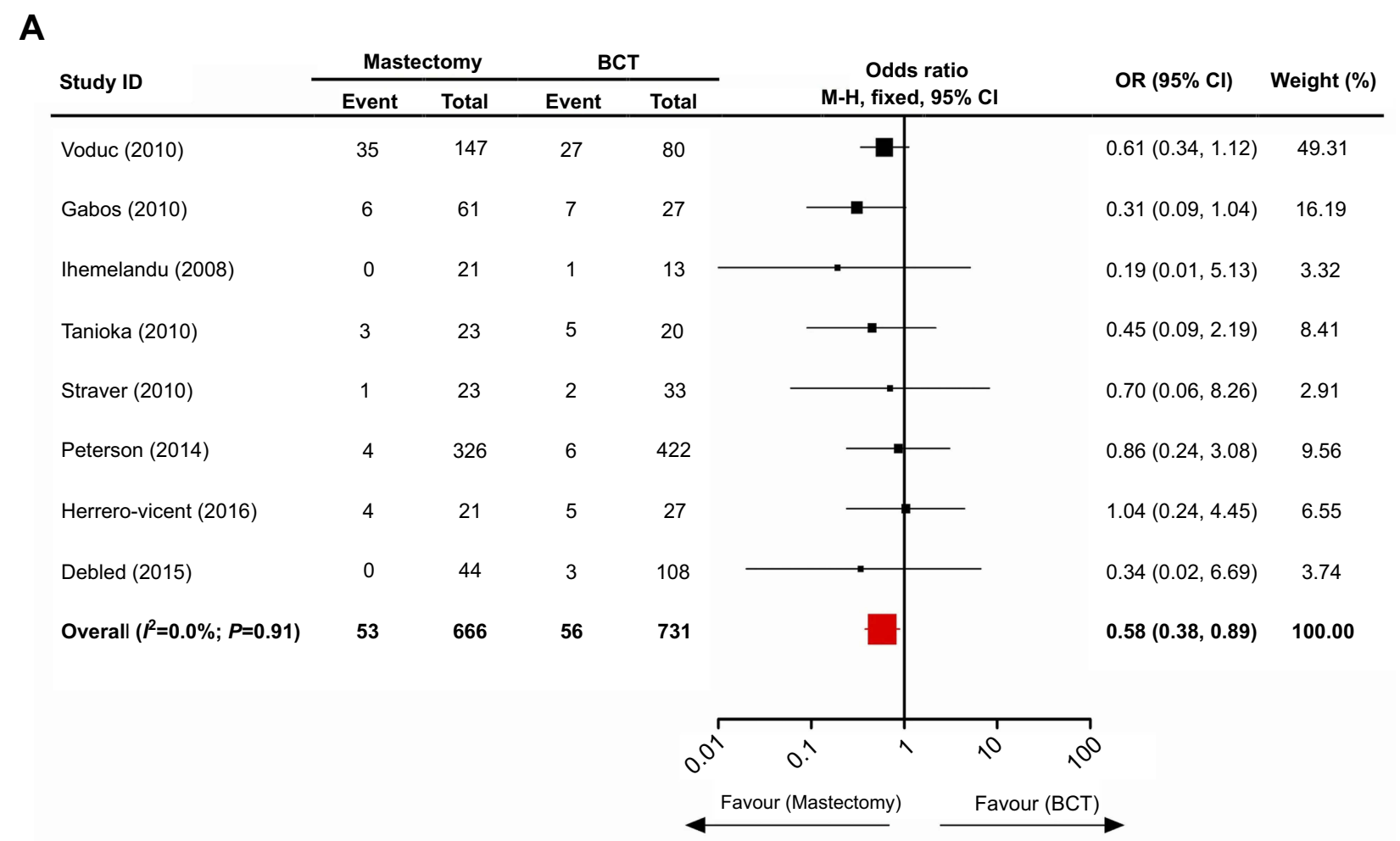

B

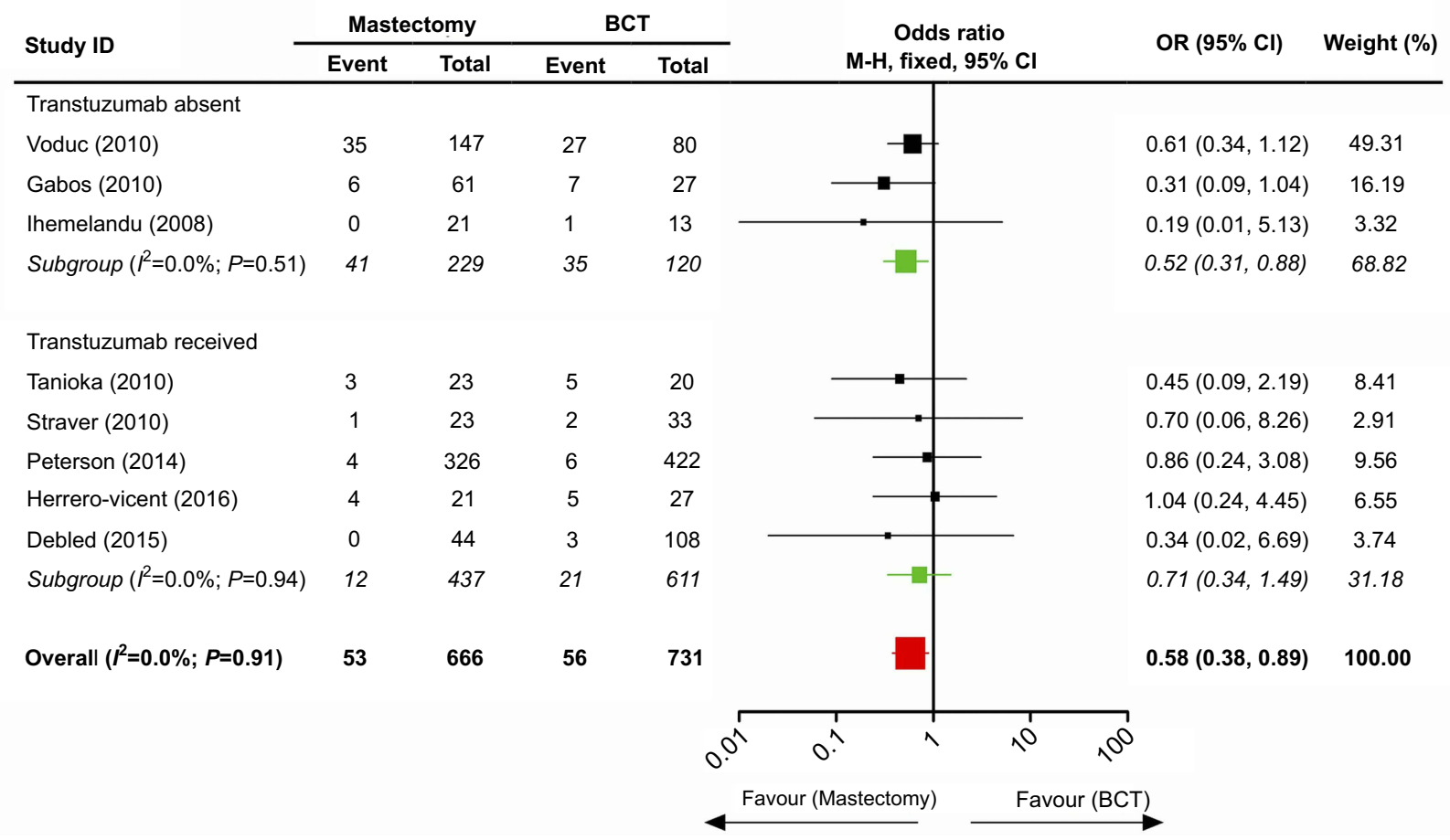

Figure 3 The comparison of local-regional relapse between mastectomy and BCT. (A) Whole group analysis; (B) Subgroup analysis. Abbreviation: $\mathrm{BCT}$, breast-conserving therapy.

partial meta-analyses were limited, which might lead to result bias. More importantly, we did not investigate other factors that might affect the outcome of LRR in women because of insufficient information provided by the publications, such as chemotherapy regimen, fractionated mode, and dose of radiotherapy. 
A

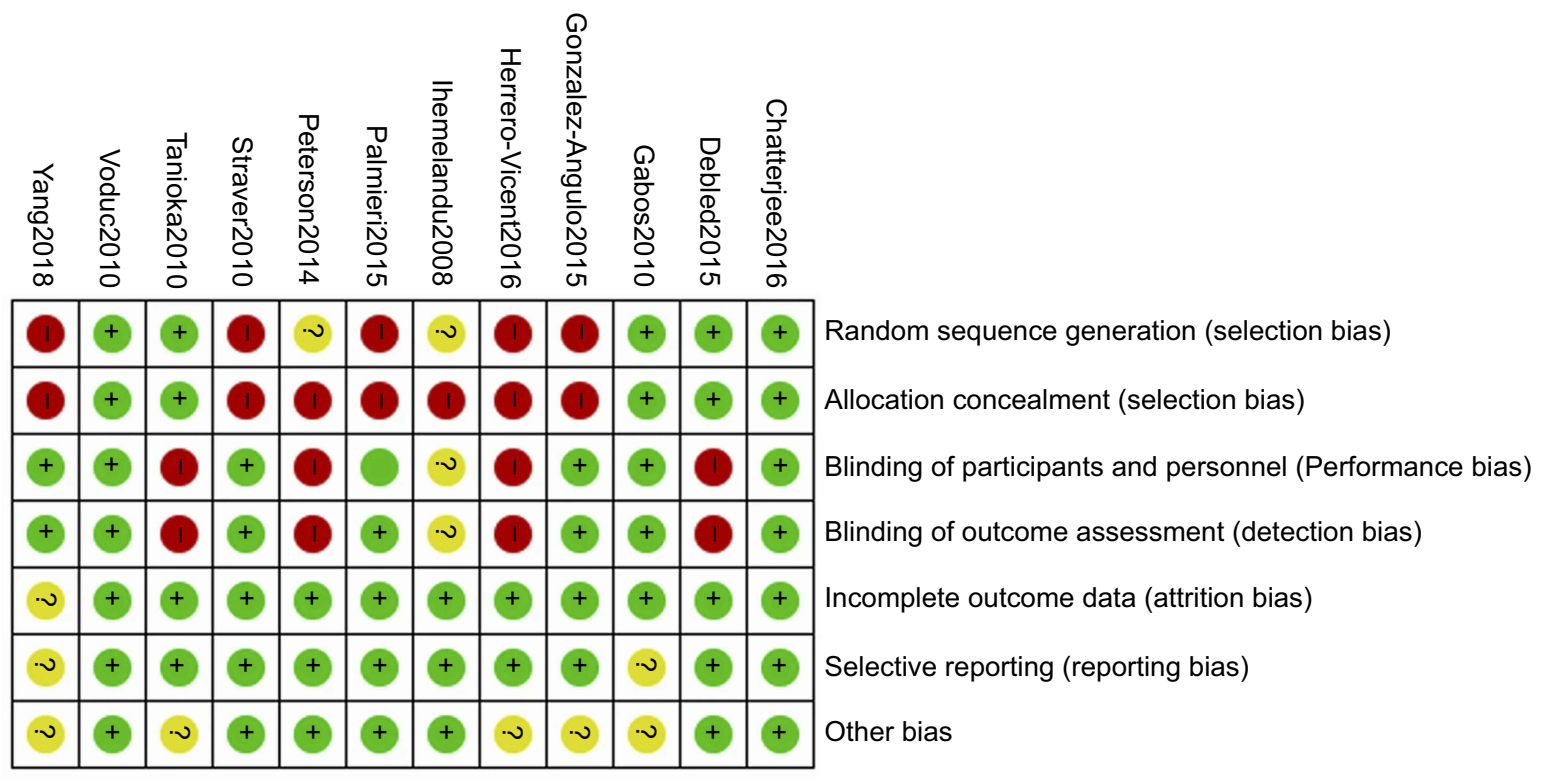

B

Random sequence generation (selection bias) Allocation concealment (selection bias) Blinding of participants and personnel (performance bias) Blinding of outcome assessment (detection bias) Incomplete outcome data (attrition bias) Selective reporting (reporting bias)

Other bias
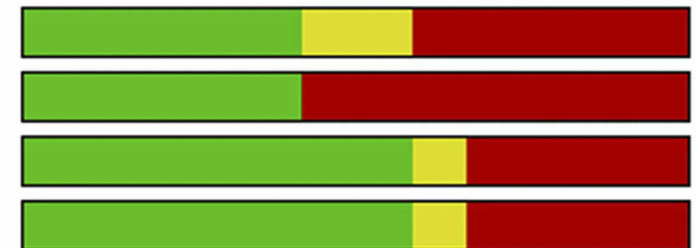

$$
\text { L }
$$

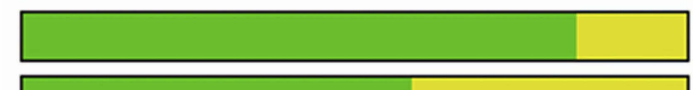

\section{Other bias}

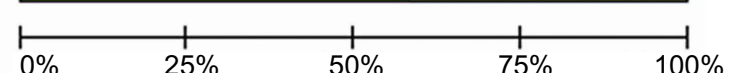

Low risk of bias

Unclear risk of bias

High risk of bias

Figure 4 The assessment of risk of bias by the new Cochrane tool. (A) The risk of bias summary; (B) The risk of bias graph.

\section{Conclusion}

The OS of HER2-amplified breast tumor patients treated with NAT is equivalent to those with AT treatment. The LRR rate of those women who undergo mastectomy compared to BCT is identical in the absence of trastuzumab treatment, but mastectomy reduces the LRR rate compared to $\mathrm{BCT}$ in women who receive trastuzumab treatment.

\section{Ethics approval and consent to participate}

This article does not contain any studies with human participants or animals performed by any of the authors.

\section{Disclosure}

The authors report no conflicts of interest in this work.

\section{References}

1. Clough KB, Acosta-Marin V, Nos C, et al. Rates of neoadjuvant chemotherapy and oncoplastic surgery for breast cancer surgery: a french national survey. Ann Surg Oncol. 2015;22(11):3504-3511. doi:10.1245/s10434-015-4378-6

2. Mougalian SS, Soulos PR, Killelea BK, et al. Use of neoadjuvant chemotherapy for patients with stage I to III breast cancer in the United States. Cancer. 2015;121(15):2544-2552. doi:10.1002/cncr.29348

3. Vugts G, Maaskant-Braat AJ, Nieuwenhuijzen GA, Roumen RM, Luiten EJ, Voogd AC. Patterns of care in the administration of neo-adjuvant chemotherapy for breast cancer. A population-based study. Breast $J$. 2016;22(3):316-321. doi:10.1111/tbj.12568 
4. Peto R, Davies C, Godwin J, et al. Comparisons between different polychemotherapy regimens for early breast cancer: meta-analyses of longterm outcome among 100,000 women in 123 randomised trials. Lancet. 2012;379(9814):432-444. doi:10.1016/S0140-6736(11)61625-5

5. Fisher B, Bryant J, Wolmark N, et al. Effect of preoperative chemotherapy on the outcome of women with operable breast cancer. $J$ Clin Oncol. 1998;16(8):2672-2685. doi:10.1200/JCO.1998.16.8.2672

6. Rastogi P, Anderson SJ, Bear HD, et al. Preoperative chemotherapy: updates of National Surgical Adjuvant Breast and Bowel Project Protocols B-18 and B-27. J Clin Oncol. 2008;26(5):778-785. doi:10.1200/JCO.2007.15.0235

7. van der Hage JA, van de Velde CJ, Julien JP, Tubiana-Hulin M, Vandervelden C, Duchateau L. Preoperative chemotherapy in primary operable breast cancer: results from the European Organization for Research and Treatment of Cancer trial 10902. J Clin Oncol. 2001;19 (22):4224-4237. doi:10.1200/JCO.2001.19.22.4224

8. Early Breast Cancer Trialist' Collaborative Group (EBCTG); Longterm outcomes for neoadjuvant versus adjuvant chemotherapy in early breast cancer: meta-analysis of individual patient data from ten randomised trials. Lancet Oncol. 2018;19(1):27-39. doi:10.1016/S1470-2045(17)30777-5

9. Mauri D, Pavlidis N, Ioannidis JP. Neoadjuvant versus adjuvant systemic treatment in breast cancer: a meta-analysis. J Natl Cancer Inst. 2005;97(3):188-194. doi:10.1093/jnci/dji021

10. Fisher B, Bauer M, Margolese R, et al. Five-year results of a randomized clinical trial comparing total mastectomy and segmental mastectomy with or without radiation in the treatment of breast cancer. $N$ Engl J Med. 1985;312(11):665-673. doi:10.1056/NEJM198503143121101

11. Veronesi U, Saccozzi R, Del Vecchio M, et al. Comparing radical mastectomy with quadrantectomy, axillary dissection, and radiotherapy in patients with small cancers of the breast. $N$ Engl J Med. 1981;305(1):6-11. doi:10.1056/NEJM198107023050102

12. Clarke M, Collins R, Darby S, et al. Effects of radiotherapy and of differences in the extent of surgery for early breast cancer on local recurrence and 15-year survival: an overview of the randomised trials. Lancet. 2005;366(9503):2087-2106. doi:10.1016/S0140-6736 (05)67887-7

13. CC N. Treatment of early breast cancer. JAMA. 1991;265:391-395.

14. Buchholz TA. Radiation therapy for early-stage breast cancer after breast-conserving surgery. $N$ Engl J Med. 2009;360(1):63-70. doi:10.1056/NEJMct0803525

15. Wolff AC, Hammond ME, Hicks DG, et al. Recommendations for human epidermal growth factor receptor 2 testing in breast cancer: American Society of Clinical Oncology/College of American Pathologists clinical practice guideline update. Arch Pathol Lab Med. 2014;138(2):241-256. doi:10.5858/arpa.2013-0953-SA

16. Voduc KD, Cheang MC, Tyldesley S, Gelmon K, Nielsen TO, Kennecke H. Breast cancer subtypes and the risk of local and regional relapse. J Clin Oncol. 2010;28(10):1684-1691. doi:10.1200/ JCO.2009.24.9284

17. Debled M, MacGrogan G, Breton-Callu C, et al. Surgery following neoadjuvant chemotherapy for HER2-positive locally advanced breast cancer. Time to reconsider the standard attitude. Eur $j$ cancer. 2015;51(6):697-704. doi:10.1016/j.ejca.2015.01.063

18. Stewart LA, Clarke M, Rovers M, et al. Preferred reporting items for systematic review and meta-analyses of individual participant data: the PRISMA-IPD statement. JAMA. 2015;313(16):1657-1665. doi:10.1001/jama.2015.3656

19. Sutton AJ, Abrams KR, Jones DR. Methods for Meta-analysis in Medical Research. Wiley Series in Probability and Statistics-applied Probability and Statistics Section. Hoboken: Wiley; 2008.

20. Ihemelandu CU, Naab TJ, Mezghebe HM, et al. Treatment and survival outcome for molecular breast cancer subtypes in black women. Ann Surg. 2008;247(3):463-469. doi:10.1097/ SLA.0b013e31815d744a
21. Gabos Z, Thoms J, Ghosh S, et al. The association between biological subtype and locoregional recurrence in newly diagnosed breast cancer. Breast Cancer Res Treat. 2010;124(1):187-194. doi:10.1007/ s10549-010-1135-1

22. Herrero-Vicent C, Guerrero-Zotano A, Gavila-Gregori J, et al. A prognostic index for locoregional recurrence after neoadjuvant chemotherapy. Ecancermedicalscience. 2016;10:647. doi:10.3332/ ecancer.2016.647

23. Straver ME, Rutgers EJ, Rodenhuis $\mathrm{S}$, et al. The relevance of breast cancer subtypes in the outcome of neoadjuvant chemotherapy. Ann Surg Oncol. 2010;17(9):2411-2418. doi:10.1245/s10434010-1008-1

24. Chatterjee S, Arunsingh M, Agrawal S, et al. Outcomes following a moderately hypofractionated adjuvant radiation (START B Type) schedule for breast cancer in an unscreened non-caucasian population. Clin Oncol ( $R$ Coll Radiol). 2016;28(10):e165-e172. doi:10.1016/j.clon.2016.05.008

25. Gonzalez-Angulo AM, Parinyanitikul N, Lei X, et al. Effect of adjuvant trastuzumab among patients treated with anti-HER2based neoadjuvant therapy. Br J Cancer. 2015;112(4):630-635. doi:10.1038/bjc.2014.647

26. Palmieri C, Macpherson IR, Yan K, et al. Neoadjuvant chemotherapy and trastuzumab versus neoadjuvant chemotherapy followed by post-operative trastuzumab for patients with HER2positive breast cancer. Oncotarget. 2016;7(11):13209-13220. doi:10.18632/oncotarget.4801

27. Lewis Phillips GD, Li G, Dugger DL, et al. Targeting HER2-positive breast cancer with trastuzumab-DM1, an antibody-cytotoxic drug conjugate. Cancer Res. 2008;68(22):9280-9290. doi:10.1158/00085472.CAN-08-1776

28. von Minckwitz G, Huang CS, Mano MS, et al. Trastuzumab emtansine for residual invasive HER2-positive breast cancer. $N$ Engl J Med. 2019;380(7):617-628. doi:10.1056/NEJMoa1814017

29. Hurvitz SA, Martin M, Symmans WF, et al. Neoadjuvant trastuzumab, pertuzumab, and chemotherapy versus trastuzumab emtansine plus pertuzumab in patients with HER2-positive breast cancer (KRISTINE): a randomised, open-label, multicentre, phase 3 trial. Lancet Oncol. 2018;19(1):115-126. doi:10.1016/S1470-2045(17) 30716-7

30. Sliwkowski MX, Lofgren JA, Lewis GD, Hotaling TE, Fendly BM, Fox JA. Nonclinical studies addressing the mechanism of action of trastuzumab (Herceptin). Semin Oncol. 1999;26(4 Suppl 12):60-70

31. Nguyen PL, Taghian AG, Katz MS, et al. Breast cancer subtype approximated by estrogen receptor, progesterone receptor, and HER-2 is associated with local and distant recurrence after breastconserving therapy. $J$ Clin Oncol. 2008;26(14):2373-2378. doi:10.1200/JCO.2007.14.4287

32. Tsoutsou PG, Vozenin MC, Durham AD, Bourhis J. How could breast cancer molecular features contribute to locoregional treatment decision making? Crit Rev Oncol Hematol. 2017;110:43-48. doi:10.1016/j.critrevonc.2016.12.006

33. Yin W, Jiang Y, Shen Z, Shao Z, Lu J. Trastuzumab in the adjuvant treatment of HER2-positive early breast cancer patients: a metaanalysis of published randomized controlled trials. PLoS One. 2011;6(6):e21030. doi:10.1371/journal.pone.0021030

34. Panoff JE, Hurley J, Takita C, et al. Risk of locoregional recurrence by receptor status in breast cancer patients receiving modern systemic therapy and post-mastectomy radiation. Breast Cancer Res Treat. 2011;128(3):899-906. doi:10.1007/s10549-011-1495-1

35. Horton JK, Jagsi R, Woodward WA, Ho A. Breast cancer biology: clinical implications for breast radiation therapy. Int $J$ Radiat Oncol Biol Phys. 2018;100(1):23-37. doi:10.1016/j.ijrobp. 2017.08 .025 


\section{Supplementary materials}

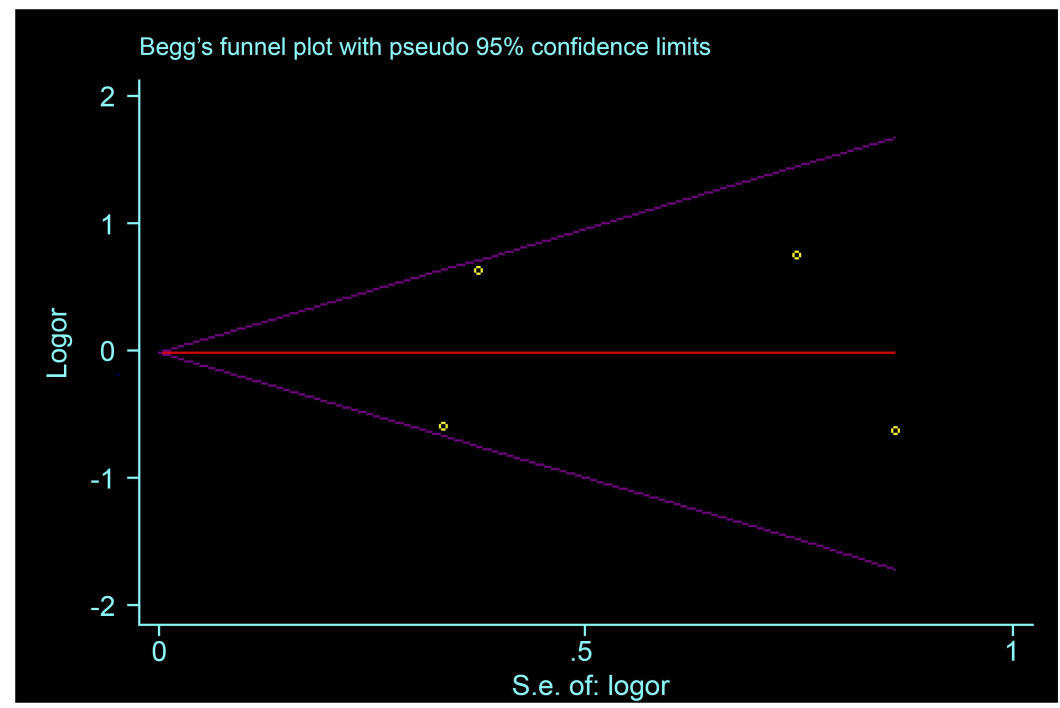

Figure SI The Begg's funnel plot with a $95 \%$ confidence interval was created to assess the presence of publication bias OS of neoadjuvant therapy versus adjuvant therapy. $p=0.844$ according to the Egg's test.

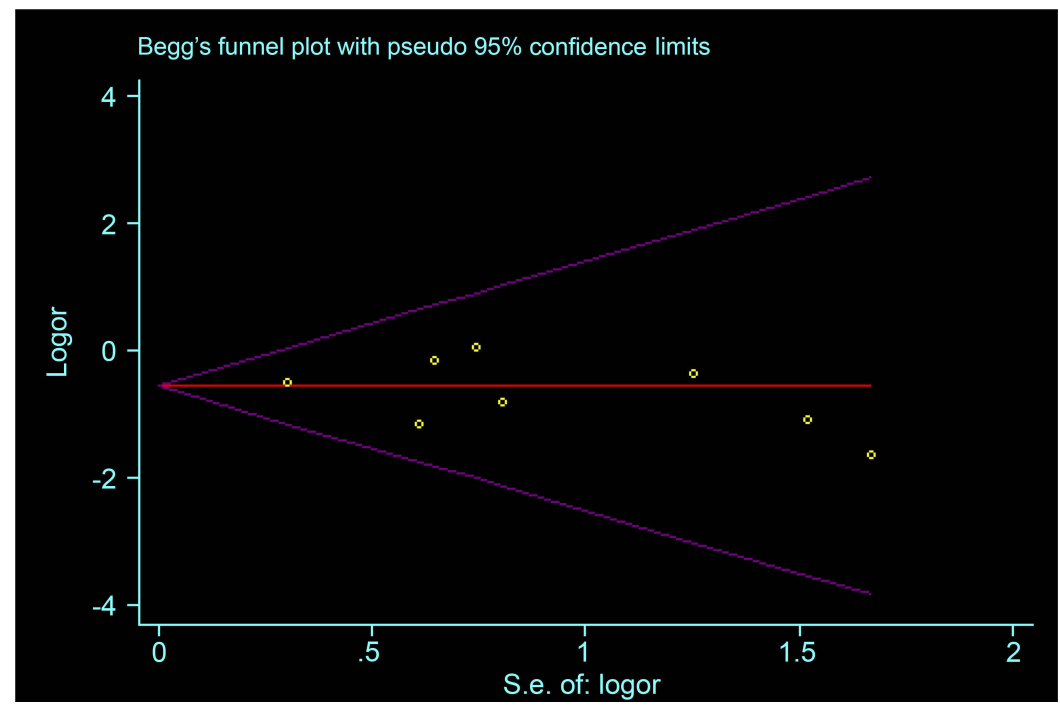

Figure S2 The Begg's funnel plot with a $95 \%$ confidence interval was created to assess the presence of publication bias in LRR of mastectomy versus breast-conserving therapy. $p=0.515$ according to the Egg's test.

\section{References}

1. Herrero-Vicent C, Guerrero-Zotano A, Gavila-Gregori J, et al.A prognostic index for locoregional recurrence after neoadjuvant chemotherapy. Ecancermedicalscience. 2016;10:647.

2. Ihemelandu CU, Naab TJ, Mezghebe HM, et al. Treatment and survival outcome for molecular breast cancer subtypes in black women. Ann Surg. 2008;247(3):463-469.

3. Gabos Z, Thoms J, Ghosh S, et al. The association between biological subtype and locoregional recurrence in newly diagnosed breast cancer. Breast Cancer Res Treat. 2010;124(1): 187-194.
4. Debled M, MacGrogan G, Breton-Callu C, et al. Surgery following neoadjuvant chemotherapy for HER2-positive locally advanced breast cancer. Time to reconsider the standard attitude. Eur $J$ Cancer. 2015;51(6):697-704.

5. Straver ME, Rutgers EJT, Rodenhuis S, et al. The relevance of breast cancer subtypes in the outcome of neoadjuvant chemotherapy. Ann Surg Oncol. 2010;17(9):2411-2418.

6. Voduc KD, Cheang MC, Tyldesley S, Gelmon K, Nielsen TO, Kennecke H. Breast cancer subtypes and the risk of local and regional relapse. J Clin Oncol. 2010;28(10):1684-1691.

7. Tanioka M, Shimizu C, Yonemori K, et al. Predictors of recurrence in breast cancer patients with a pathologic complete response after neoadjuvant chemotherapy. Br J Cancer. 2010;103(3):297-302. 
8. Peterson DJ, Truong PT, Sadek BT, et al. Locoregional recurrence and survival outcomes by type of local therapy and trastuzumab use among women with node-negative, HER2-positive breast cancer. Ann Surg Oncol. 2014;21(11):3490-3496.

9. Chatterjee S, Arunsingh M, Agrawal S, et al. Outcomes following a moderately hypofractionated adjuvant radiation (START B Type) schedule for breast cancer in an unscreened non-caucasian population. Clin Oncol. 2016;28(10):e165-e172.

10. Gonzalez-Angulo AM, Parinyanitikul N, Lei X, et al Effect of adjuvant trastuzumab among patients treated with anti-HER2-based neoadjuvant therapy. Br J Cancer. 2015;112(4):630-635.
11. Yang H, Zhou L, Wang S, et al. Retrospective analysis of concurrent docetaxel and epirubicin neoadjuvant versus adjuvant chemotherapy: Which leads to better outcomes for different subtype breast cancer patients? Medicine. 2018;97(40):e12690.

12. Palmieri C, Macpherson IR, Yan K, et al. Neoadjuvant chemotherapy and trastuzumab versus neoadjuvant chemotherapy followed by postoperative trastuzumab for patients with HER2-positive breast cancer. Oncotarget. 2016;7(11):13209-13220. 


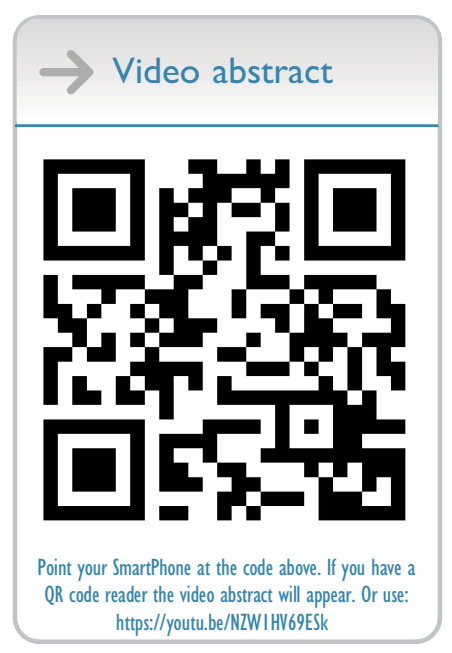

\section{Publish your work in this journal}

Cancer Management and Research is an international, peer-reviewed open access journal focusing on cancer research and the optimal use of preventative and integrated treatment interventions to achieve improved outcomes, enhanced survival and quality of life for the cancer patient.
The manuscript management system is completely online and includes a very quick and fair peer-review system, which is all easy to use. Visit http://www.dovepress.com/testimonials.php to read real quotes from published authors. 